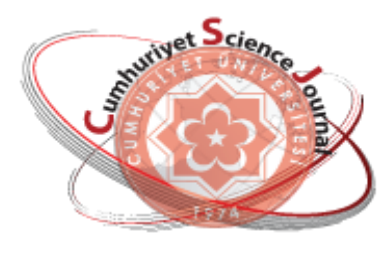

e-ISSN: $2587-246 X$

ISSN: $2587-2680$

\section{Cumburiyet Seionee Journal esy}

Cumhuriyet Sci. J., Vol.40-2(2019) 285-298

\title{
Analysis of a Random Zeeman Heartbeat Model with Differential Transformation Method
}

\author{
PInar ORAL ${ }^{1(D)}$, Mehmet MERDAN ${ }^{*}$ (D), Zafer BEKIRYAZICI ${ }^{2}$ (D) \\ ${ }^{1}$ Department of Mathematical Engineering, Gumushane University, Gumushane, TURKEY \\ ${ }^{2}$ Department of Mathematics, Recep Tayyip Erdogan University, Rize, TURKEY
}

\begin{abstract}
In this paper, the differential transformation method is used to examine the random Zeeman Heartbeat Model. Some of the parameters and the initial conditions of the model are taken as random variables with Beta and Normal distributions, respectively. The approximate analytical solution of the random Zeeman Model is obtained and used to find the expectation and variance of the model components. The results from the random models including Beta and normal distributed random effects are compared and the approximate numerical characteristics are obtained for these cases. The approximate formulas are also modified by using Laplace-Padé Method to increase the convergence interval of the approximations.
\end{abstract}

Keywords: Zeeman Heartbeat Model, Random differential equation, Expected value, Variance, Padé approximation.

\section{Diferansiyel Dönüşüm Yöntemi İle Rastgele Kalp Atış Modelinin Analizi}

Özet. Bu çalışmada, rastgele Zeeman Kalpatış Modelinin incelenmesi için diferansiyel dönüşüm yöntemi kullanılmıştır. Modelin bazı parametreleri ve başlangıç koşulları sırasıyla Beta ve Normal dağılımlara sahip rastgele değişkenler olarak alınmıştır. Rastgele Zeeman Modelinin yaklaşık analitik çözümü elde edilmiş ve model bileşenlerinin beklenen değer ve varyansı elde edilmiştir. Beta ve normal olarak dağılmış rastgele etkiler altında, rastgele modellerin sonuçları karşılaştırılmış ve bu durumlar için elde edilen yaklaşık sayısal karakteristikler karşılaştırılmıştır. Elde edilen yaklaşık formüllere, yaklaşımların yakınsama aralığını artırmak için Laplace-Padé Metodu uygulanarak iyileştirilmiş çözümler bulunmuştur.

Anahtar Kelimeler: Zeeman Kalpatış Modeli, Rastgele diferansiyel denklem, Beklenen değer, Varyans, Padé yaklaşımı

\section{INTRODUCTION}

The majority of the investigations of mathematical models in various fields of science is performed by using deterministic components, ignoring the randomness of the natural course of events. This fact is a setback for an accurate description of the events through equation systems since there is always an unignorable amount of uncertainty in the natural realizations of all phenomena. The random effects approach of Merdan et al. aims to handle this issue swiftly by introducing random noise terms in the deterministic parameters of compartmental mathematical models [1,2]. Compartmental models are widely used in the modeling of infectious diseases and such a random modeling approach provides a straightforward modification of these models to effectively describe the random behavior of diseases. 
The model used in this study has been introduced by E.C. Zeeman in 1972 [3-5]. It consists of differential equations that describe the heart activity under the control of electrical impulses produced by the body. The heart regulates the circulation of blood in the body through the contraction of heart muscles, an event which is triggered by an electrochemical activity. The equation systems of E.C. Zeeman represent the dynamics of the muscle fiber activity and stimulus which controls muscle fiber contraction over time. The parameters of the differential equation system will be added random effects with Gaussian (normal) and beta distribution to represent the natural fluctuations in the dynamics of heartbeat. Similar modeling studies have been made with random effects for bacterial resistance and biochemical reactions $[2,6]$.

In this study, Differential Transformation Method (DTM) will be used to investigate the Zeeman's heartbeat models with random components. DTM $[7,8]$ and similar methods related to DTM have been used for analyzing various mathematical models $[17,18]$. While DTM has many advantages for analyzing models such its adaptability for use in fractional, delay and partial differential equation systems, its power series form approximate-analytical solution which enables a detailed analysis of the problem of interest and its usability in many problems in applied mathematics. However, the series solution brings along a serious disadvantage as well, which arises when the problems are analyzed in wide time intervals, causing issues on the convergence of the approximate solution. One of the techniques for tackling this problem is the Laplace-Pade method, which proposes the use of a ratio two polynomial functions of orders $p$ and $q$, where the orders are arbitrary positive integers. Applications have shown that every different selection of $p, q$ results in a new approximate solution and generally the selection for $p=q$ provides the best convergence. Modifying the approximate analytical solution through Pade approximants provides a new approximate solution which is convergent to the exact solution on a wider time interval. Details of Laplace-Pade modification of DTM have been given in the literature [19-20].

Our study concentrates on the improvement of the approximate solutions of the Zeeman heartbeat model with random components obtained with DTM through Laplace-Pade method [18, 21]. The paper is organized as follows: Section 2 includes an analysis of the heartbeat model with two equations including an introduction of the Differential Transformation Method and the distributions used. Section 3 contains the analysis on the model with three equations. The last section contains the conclusions.

\section{TWO DIMENSIONAL ZEEMAN MODEL}

The Zeeman model is used for the modeling of heartbeat and the two dimensional version consists of the following differential equations, where $x_{1}$ represents the heart muscle fiber length and $x_{2}$ represents the stimulus:

$$
\begin{aligned}
& \frac{d x_{1}}{d t}=-\frac{1}{\epsilon}\left(x_{1}^{3}-T x_{1}+x_{2}\right), \\
& \frac{d x_{2}}{d t}=x_{1}-x_{d} .
\end{aligned}
$$

The parameters of this system, $\epsilon, T, x_{d}$ are defined as follows: $T$ represents the tension $(T>0), \epsilon$ is a constant that depends on the timescale and $x_{d}$ is the typical relaxed fiber length. The deterministic values of the parameters have been given as $\epsilon=0.2 ; T=0.5$ and $x_{d}=0$ or 0.41 whereas the initial values of (1) are given as $x_{1}(0)=0.5$ and $x_{2}(0)=0[3-5]$.

\subsection{Differential Transformation Method}


An outline of the Differential Transformation Method (DTM) can be given as follows. Let $x(t)$ be an analytical function in the domain $D$ and $t=t_{0}$ be a point in $D$. The function $x(t)$ can be represented by using a $t_{0}$-centered power series. The $\mathrm{k}$-th derivative of $x(t)$ is defined as:

$$
X(k)=\frac{1}{k !}\left[\frac{d^{k} x(t)}{d t^{k}}\right]_{t=t_{0}}, \forall t \in D
$$

In (2), $x(t)$ is the original function and $X(k)$ is the transformed function. The inverse differential transformation of $X(k)$ is given as [7,8]:

$$
x(t)=\sum_{k=0}^{\infty} X(k)\left(t-t_{0}\right)^{k}, \forall t \in D .
$$

Using (2) and (3), we obtain

$$
x(t)=\sum_{k=0}^{\infty} \frac{\left(t-t_{0}\right)^{k}}{k !}\left[\frac{d^{k} x(t)}{d t^{k}}\right]_{t=t_{0}}, \forall t \in D
$$

The basic theorems for one-dimensional differential transformation are given as below.

Theorem 1. If $z(t)=x(t) \pm y(t)$, then $Z(k)=X(k) \pm Y(k)$.

Theorem 2. If $z(t)=c y(t)$, then $Z(k)=c Y(k)$.

Theorem 3. If $z(t)=\frac{d y(t)}{d t}$, then $Z(k)=(\mathrm{k}+1) Y(k+1)$.

Theorem 4. If $Z(t)=\frac{d^{n} y(t)}{d t}$, then $Z(k)=\frac{(\mathrm{k}+\mathrm{n}) !}{k !} Y(k+n)$.

Theorem 5. If $z(t)=x(t) y(t), Z(k)=\sum_{k_{1}=0}^{k} X\left(k_{1}\right) Y\left(k-k_{1}\right)$.

Theorem 6. If $z(t)=t^{n}$, then $Z(k)=\delta(k-n)=\left\{\begin{array}{ll}1 & k=n \\ 0 & k \neq n\end{array}\right.$.

In applications, the function $x(t)$ is represented by an infinite series and by using (4) we can write:

$$
x(t)=\sum_{k=0}^{N} X(k)\left(t-t_{0}\right)^{k}, \forall t \in D .
$$

The equation (5) states that $\sum_{k=N+1}^{\infty} X(k)\left(t-t_{0}\right)^{k}$ has a negligibly small value.

Consider a system of ordinary differential equations

$$
\begin{aligned}
& \frac{d x_{1}}{d t}+h_{1}\left(t, x_{1}, x_{2}, \ldots, x_{m}\right)=g_{1}(t), \\
& \frac{d x_{2}}{d t}+h_{2}\left(t, x_{1}, x_{2}, \ldots, x_{m}\right)=g_{2}(t), \\
& \frac{d x_{m}}{d t}+h_{m}\left(t, x_{1}, x_{2}, \ldots, x_{m}\right)=g_{m}(t),
\end{aligned}
$$

with the initial values

$$
x_{1}\left(t_{0}\right)=d_{1}, \quad x_{2}\left(t_{0}\right)=d_{2}, \cdots, x_{m}\left(t_{0}\right)=d_{m}
$$


Using DTM, the derivative of both sides in the equation system (6)-(7) is obtained as

$$
\begin{aligned}
& (k+1) X_{1}(k+1)+H_{1}(k)=G_{1}(k), \\
& (k+1) X_{2}(k+1)+H_{2}(k)=G_{2}(k),
\end{aligned}
$$

$\vdots$

$$
(k+1) X_{m}(k+1)+H_{m}(k)=G_{m}(k) .
$$

with

$$
X_{1}(0)=d_{1}, \quad X_{2}(0)=d_{2}, \cdots, X_{m}(0)=d_{m} .
$$

Similarly, the $\mathrm{n}$-th term is obtained for (5) as

$$
\begin{aligned}
& \varphi_{1, n}(t)=x_{1}(t)=\sum_{k=1}^{N} X_{1}(k) t^{k}, \\
& \varphi_{2, n}(t)=x_{2}(t)=\sum_{k=1}^{N} X_{2}(k) t^{k},
\end{aligned}
$$

$\vdots$

$$
\varphi_{m, n}(t)=x_{m}(t)=\sum_{k=1}^{N} X_{m}(k) t^{k}
$$

Using DTM for equation (1), we obtain:

$$
\begin{aligned}
& (k+1) X_{1}(k+1)=-\frac{1}{\varepsilon}\left[\sum_{k_{2}=0}^{k} \sum_{k_{1}=0}^{k_{2}} X_{1}\left(k_{1}\right) X_{1}\left(k_{2}-k_{1}\right) X_{1}\left(k-k_{2}\right)-T X_{1}(k)+X_{2}(k)\right], \\
& (k+1) X_{2}(k+1)=X_{1}(k)-x_{d} \delta(k) .
\end{aligned}
$$

We will be choosing random parameters and initial values for the two dimensional Zeeman model to obtain a random model. In the next section, we introduce the beta and normal distributions which we will be using for these random components.

\subsection{Beta Distribution}

The Beta function $B(\alpha, \beta)$ for the parameters $>0, \beta>0$ is defined as

$$
B(\alpha, \beta)=\int_{0}^{1} x^{\alpha-1}(1-x)^{\beta-1} d x .
$$

Dividing both sides of $(12)$ by $B(\alpha, \beta)$, we obtain

$$
1=\frac{1}{B(\alpha, \beta)} \int_{0}^{1} x^{\alpha-1}(1-x)^{\beta-1} d x .
$$

Using the definition of a probability density function, we obtain [9]: 
where

$$
f(x)=\left\{\begin{array}{c}
\frac{x^{\alpha-1}(1-x)^{\beta-1}}{B(\alpha, \beta)}, 0<x<1 . \\
0, \quad \text { elsewhere }
\end{array}\right.
$$

$$
B(\alpha, \beta)=\frac{\Gamma(\alpha) \cdot \Gamma(\beta)}{\Gamma(\alpha+\beta)} .
$$

If $X \sim B(\alpha, \beta)$ is a Beta distributed random variable then,

$$
\begin{gathered}
E[X]=\frac{\alpha}{\alpha+\beta}, \quad \operatorname{Var}[X]=\frac{\alpha \beta}{(\alpha+\beta)^{2} \cdot(\alpha+\beta+1)}, \\
E\left[X^{2}\right]=\frac{\alpha(\alpha+1)}{(\alpha+\beta+1)(\alpha+\beta)}, \quad E\left[X^{3}\right]=\frac{\alpha(\alpha+1)(\alpha+2)}{(\alpha+\beta+2)(\alpha+\beta+1)(\alpha+\beta)}, \ldots \\
E\left[X^{N}\right]=\frac{\alpha(\alpha+1)(\alpha+2) \ldots(\alpha+(N-1))}{(\alpha+\beta+N-1)(\alpha+\beta+N-2) \ldots(\alpha+\beta+1)(\alpha+\beta)} .
\end{gathered}
$$

\subsection{Expected Value of the Two Dimensional Zeeman Model}

Using Differential Transformation Method [7,8] and its random counterpart [10-14], we can find the approximate solutions of a random process in the form of a power series by using the formula

$$
x_{N}(t)=\sum_{k=0}^{N} X(k) t^{k}
$$

The variance and covariance for the approximate solution $x_{N}(t)$ are given as

where [11]

$$
\begin{gathered}
E\left[x_{N}(t)\right]=\sum_{k=0}^{N} E[X(k)] t^{k}, \\
\operatorname{Var}\left[x_{N}(t)\right]=\sum_{j=0}^{N} \sum_{i=0}^{N} \operatorname{cov}(X(i), X(j)) t^{i+j},
\end{gathered}
$$

$$
\operatorname{cov}(X(i), X(j))=E(X(i) X(j))-E[X(i)] E[X(j)], \quad \forall i, j=0,1, \ldots, N .
$$

Let the parameter $T$ in (1) be a Beta distributed random variable such that $T \sim \operatorname{Beta}(\alpha=2, \beta=1)$. In addition, assume that the initial conditions are assumed to be random variables $Y_{0}$ and $Y_{1}$ where $Y_{0}$ and $Y_{1}$ are independent random variables with normal distribution, i.e. $Y_{0}, Y_{1} \in N\left(\mu, \sigma^{2}\right)$. Let $\mu=1, \sigma^{2}=1, \sigma=$ 1. Then,

$$
\begin{aligned}
& E\left[Y_{0}\right]=\mu=1, E\left[Y_{1}\right]=\mu=1 \\
& E\left[Y_{0}^{2}\right]=\mu^{2}+\sigma^{2}=1+1=2, \\
& E\left[Y_{1}^{2}\right]=\mu^{2}+\sigma^{2}=1+1=2 \\
& E\left[Y_{0}^{3}\right]=E\left[Y_{1}^{3}\right]=\mu^{3}+3 \mu \sigma^{2}=1+3.1 .1=4 \\
& E\left[Y_{0}^{4}\right]=E\left[Y_{1}^{4}\right]=\mu^{3}+6 \mu^{2} \sigma^{2}+3 \sigma^{4}=1+6.1 .1+3.1=10 \\
& E\left[Y_{0}^{5}\right]=E\left[Y_{1}^{5}\right]=\mu^{5}+10 \mu^{3} \sigma^{2}+15 \mu \sigma^{4}=1+10.1 .1+15.1=26, \\
& E\left[Y_{0}^{6}\right]=E\left[Y_{1}^{6}\right]=\mu^{6}+15 \mu^{4} \sigma^{2}+45 \mu^{2} \sigma^{4}+15 \sigma^{6}=1+15.1 .+45.1 .1+15.1=76, \\
& E\left[Y_{0}^{7}\right]=E\left[Y_{1}^{7}\right]=\mu^{7}+21 \mu^{5} \sigma^{2}+105 \mu^{3} \sigma^{4}+105 \mu^{3} \sigma^{6}=232 .
\end{aligned}
$$


Since $T>0$ is Beta distributed such that $T \sim B(\alpha=2, \beta=1)$ [9],

$$
\begin{aligned}
& E[T]=\frac{\alpha}{\alpha+\beta}=\frac{2}{3} \\
& E\left[T^{2}\right]=\frac{\alpha(\alpha+1)}{(\alpha+\beta+1)(\alpha+\beta)}=\frac{2.3}{3.4}=\frac{1}{2} \\
& E\left[T^{3}\right]=\frac{\alpha(\alpha+1)(\alpha+2)}{(\alpha+\beta+2)(\alpha+\beta+1)(\alpha+\beta)}=\frac{2}{5} \\
& E\left[T^{4}\right]=\frac{\alpha(\alpha+1)(\alpha+2)(\alpha+3)}{(\alpha+\beta)(\alpha+\beta+1)(\alpha+\beta+2)(\alpha+\beta+3)}=\frac{1}{3} .
\end{aligned}
$$

Using (11), the approximate analytical solution of $x_{1}(t)$ in (1) using 4 terms is obtained as (denoted by $\left.X_{1}\right)$

$$
\begin{aligned}
X 1=Y_{0}+( & \left.-5 Y_{1}+5 T Y_{0}-5 Y_{0}^{3}\right) t+\left(\frac{75}{2} Y_{0}^{5}-50 Y_{0}^{3} T+\frac{75}{2} Y_{0}^{2} Y_{1}+\frac{25}{2} Y_{0} T^{2}-\frac{25}{2} Y_{1} T-\frac{5}{2} Y_{0}\right) t^{2} \\
& +\left(\frac{25}{6} Y_{1}+\frac{50}{3} Y_{0}^{3}-\frac{625}{2} Y_{0}^{7}+\frac{125}{6} Y_{0} T^{3}-\frac{125}{6} Y_{1} T^{2}+\frac{1125}{2} Y_{0}^{5} T-\frac{875}{2} Y_{0}^{4} Y_{1}\right. \\
& \left.-\frac{1625}{6} Y_{0}^{3} T^{2}-125 Y_{0} Y_{1}^{2}+375 Y_{0}^{2} Y_{1} T-\frac{25}{3} Y_{0} T\right) t^{3}+\cdots
\end{aligned}
$$

Hence, the approximate expected value of the random variable $X_{1}$ is obtained as

$$
\begin{aligned}
E[X 1]=E\left[Y_{0}\right. & +\left(-5 Y_{1}+5 T Y_{0}-5 Y_{0}^{3}\right) t \\
& +\left(\frac{75}{2} Y_{0}^{5}-50 Y_{0}^{3} T+\frac{75}{2} Y_{0}^{2} Y_{1}+\frac{25}{2} Y_{0} T^{2}-\frac{25}{2} Y_{1} T-\frac{5}{2} Y_{0}\right) t^{2} \\
& +\left(\frac{25}{6} Y_{1}+\frac{50}{3} Y_{0}^{3}-\frac{625}{2} Y_{0}^{7}+\frac{125}{6} Y_{0} T^{3}-\frac{125}{6} Y_{1} T^{2}+\frac{1125}{2} Y_{0}^{5} T-\frac{875}{2} Y_{0}^{4} Y_{1}\right. \\
& \left.\left.-\frac{1625}{6} Y_{0}^{3} T^{2}-125 Y_{0} Y_{1}^{2}+375 Y_{0}^{2} Y_{1} T-\frac{25}{3} Y_{0} T\right) t^{3}\right]+\cdots
\end{aligned}
$$

Using the independence of the random variables, we find

$$
E\left[X_{1}\right]=1-\frac{65}{3} t+\frac{10945}{12} t^{2}-67353.472 t^{3}+\cdots
$$

The expected value of $X_{1}$ is shown in Figure 1 . 


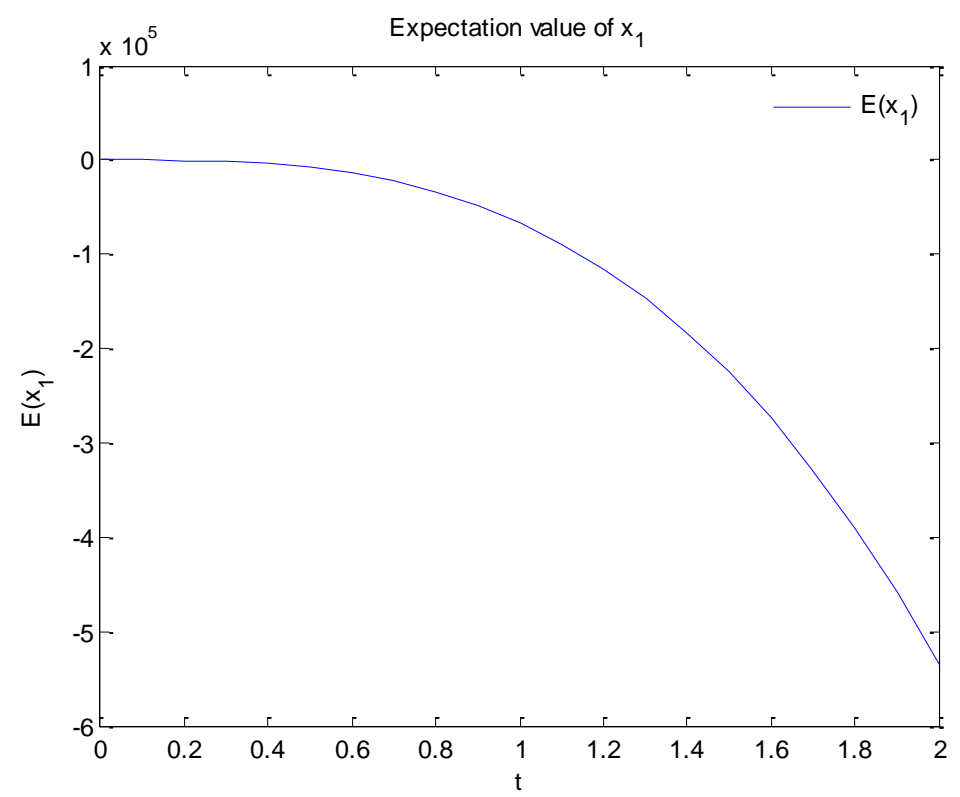

Figure 1. The expected value of $X_{1}$.

$$
\begin{aligned}
X_{2}=Y_{1}+Y_{0} t & +\left(-\frac{5}{2} Y_{1}+\frac{5}{2} T Y_{0}-\frac{5}{2} Y_{0}^{3}\right) t^{2} \\
& +\left(\frac{25}{2} Y_{0}^{5}-\frac{50}{3} Y_{0}^{3} T+\frac{25}{2} Y_{0}^{2} Y_{1}+\frac{25}{6} Y_{0} T^{2}-\frac{25}{6} Y_{1} T-\frac{5}{6} Y_{0}\right) t^{3}+\cdots
\end{aligned}
$$

Hence, its expectation becomes

$$
\begin{aligned}
E\left[X_{2}\right]=E\left[Y_{1}+\right. & Y_{0} t+\left(-\frac{5}{2} Y_{1}+\frac{5}{2} T Y_{0}-\frac{5}{2} Y_{0}^{3}\right) t^{2} \\
& \left.+\left(\frac{25}{2} Y_{0}^{5}-\frac{50}{3} Y_{0}^{3} T+\frac{25}{2} Y_{0}^{2} Y_{1}+\frac{25}{6} Y_{0} T^{2}-\frac{25}{6} Y_{1} T-\frac{5}{6} Y_{0}\right) t^{3}\right]+\cdots \\
E\left[X_{2}\right]=1+t & -\frac{65}{6} t^{2}+\frac{10945}{36} t^{3}+\cdots
\end{aligned}
$$

The approximate expected value of $X_{2}$ is shown in Figure 2. It should be noted that more terms are needed for accurate approximate expectations $E\left[X_{1}\right], E\left[X_{2}\right]$. The first three terms have been given to present the calculation method and to underline the improvements obtained by the modification. 


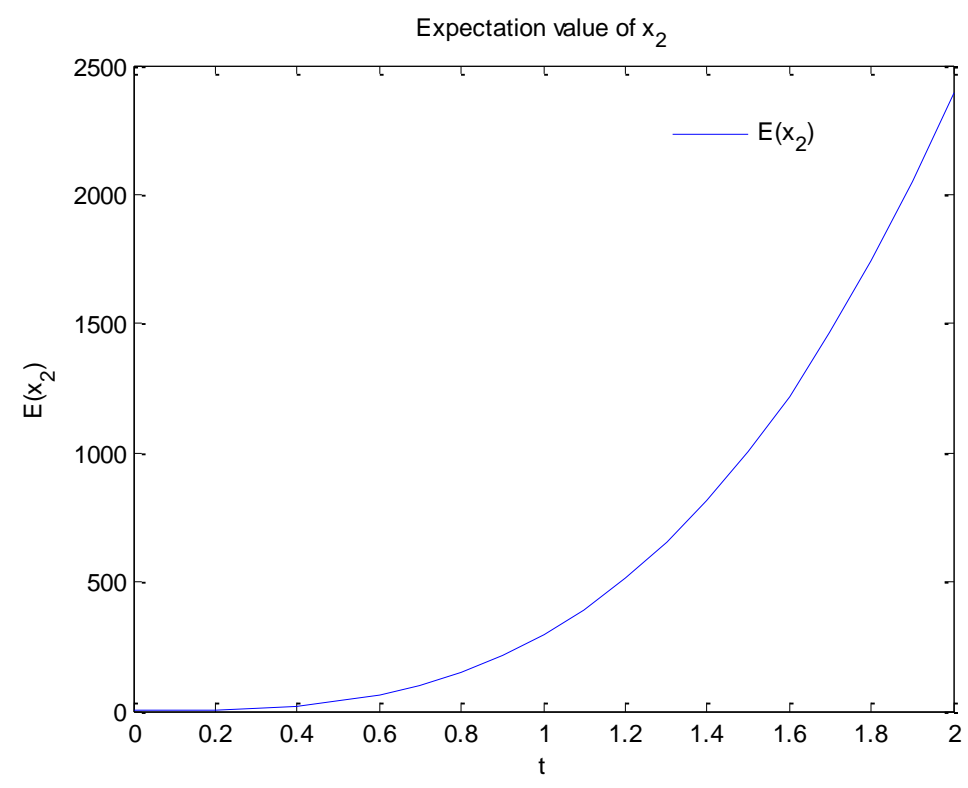

Figure 2. The expected value of $\mathrm{X}_{2}$.

\subsection{Laplace-Padé Method}

If Laplace-Padé method $[15,16]$ is used to improve the results, i.e. to obtain convergent results in a wider interval, the following result is obtained for (21):

$$
\begin{aligned}
& M_{1} \\
& =\frac{1}{7424222958497731096191780362649627} e^{-\frac{56373593243647997}{418931110051840} t} \\
& \times(7424222958497731096191780362649627 \\
& \times \cosh \left(\frac{1}{418931110051840} t \sqrt{2474740986165910365397260120883209}\right) \\
& +141890257577574391 \sqrt{2474740986165910365397260120883209} \\
& \left.\times \sinh \left(\frac{1}{418931110051840} t \sqrt{2474740986165910365397260120883209}\right)\right)
\end{aligned}
$$

This new expected value of $X_{1}$, denoted by $M 1$, is shown in Figure 3 .

Similarly, Laplace-Padé method for (22) gives:

$$
\begin{array}{r}
M_{2}=\frac{1}{381232315} e^{-\frac{11075}{272} t}\left(381232315 \cosh \left(\frac{1}{816} t \sqrt{1143696945}\right)\right. \\
\left.+11347 \sqrt{1143696945} \sinh \left(\frac{1}{816} t \sqrt{1143696945}\right)\right)
\end{array}
$$

The modified expected value for $X_{2}$, denoted by $M_{2}$, is shown in Figure 4. 


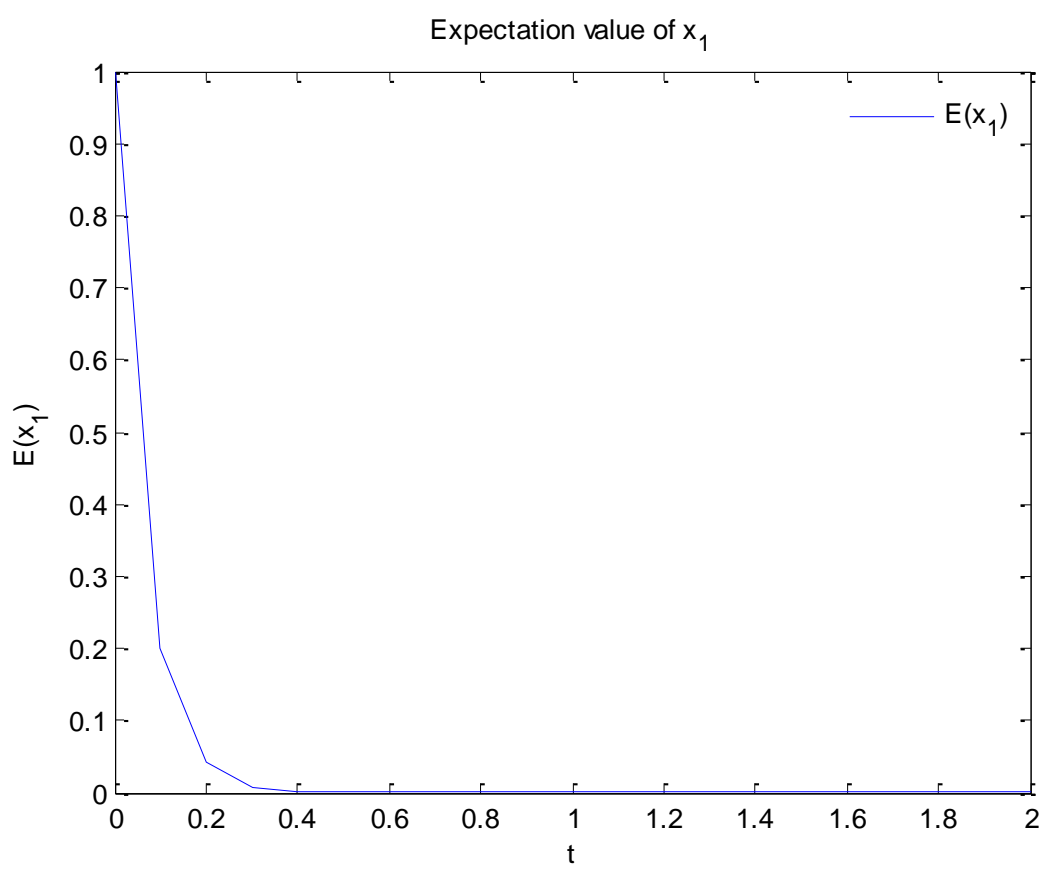

Figure 3. The expected value of $X_{1}$, obtained by modified DTM.

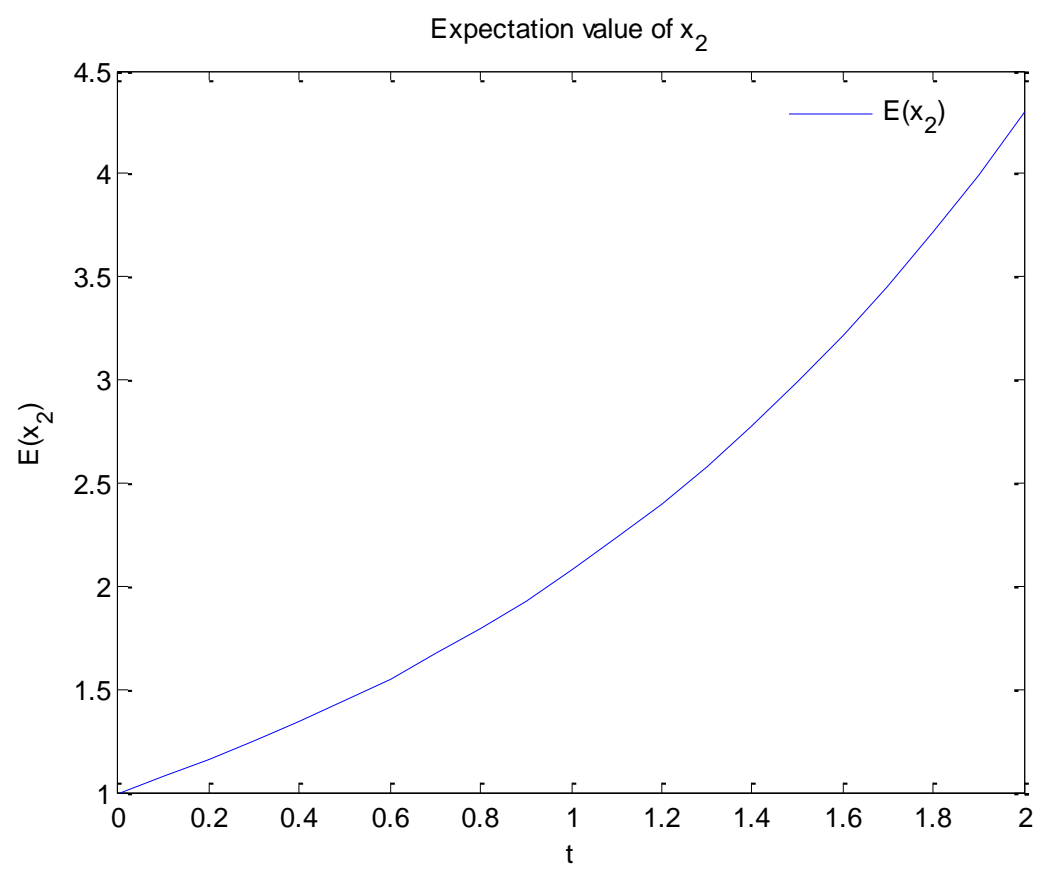

Figure 4. The expected value of $X_{2}$, obtained by modified DTM.

\subsection{Variances for Two Dimensional Zeeman Model}

Using the formulas (17) and (18), the variances for the two dimensional model is obtained as follows.

For the variance of $x_{1}$ (using a three term truncated approximation): 


$$
\operatorname{Var}\left[X_{1}(t)\right]=\sum_{j=0}^{2} \sum_{i=0}^{2} \operatorname{cov}\left(X_{1}(i), X_{1}(j)\right) t^{i+j}=1-\frac{160}{3} t+\frac{87235}{18} t^{2}+\cdots
$$

Laplace-Padé method for (25) gives

$$
\begin{gathered}
M_{3}=-\frac{1}{56738917631} e^{\left(\frac{1395760}{36981} t\right)\left(-56738917631 \cosh \left(\frac{73}{36981} t \sqrt{3886227235}\right)\right.} \\
\left.+673616 \sqrt{3886227235} \sinh \left(\frac{73}{36981} t \sqrt{3886227235}\right)\right)
\end{gathered}
$$

Similarly, the variance for $X_{2}$ is:

$$
\operatorname{Var}\left[X_{2}(t)\right]=\sum_{j=0}^{2} \sum_{i=0}^{2} \operatorname{cov}\left(X_{1}(i), X_{1}(j)\right) t^{i+j}=1-4 t^{2}+\cdots
$$

and the modified variance becomes

$$
M_{4}=\cos (2 \sqrt{2} t)
$$

\subsection{Expected Values for Initial Conditions with Normal Distribution}

Assume that the initial conditions $Y_{0}$ and $Y_{1}$ of (1) are independent random variables with normal distribution. Using (19), the expected value of the (truncated) approximate solution $X_{1}$ becomes (note that unlike Section 2.3, the parameter $T$ is not a random variable here):

$$
E\left[X_{1}\right]=1-\frac{45}{2} t+\frac{7555}{8} t^{2}-69644.271 t^{3}+\cdots
$$

If Laplace-Padé method is applied to (25), we obtain

$$
\begin{aligned}
M_{5}=0.1 \times 10^{-9} & (9999999999 \cosh (119.2044503 t) \\
& +9501091052 \sinh (119.2044503 t)) e^{-135.7572336 t} .
\end{aligned}
$$

Similarly, the expected value of the approximate solution $X_{2}$ is

$$
E\left[X_{2}\right]=1+t-\frac{45}{4} t^{2}+\frac{7555}{24} t^{3}+\cdots
$$

Its modification is obtained as

$$
\begin{array}{r}
M_{6}=\frac{1}{60525305} e^{\left(-\frac{7645}{188} t\right)}\left(60525305 \cosh \left(\frac{1}{188} t \sqrt{60525305}\right)\right. \\
\left.+7833 \sqrt{60525305} \sinh \left(\frac{1}{188} t \sqrt{60525305}\right)\right)
\end{array}
$$




\subsection{Expected Values for Random $T$ Parameter with Beta Distribution}

If the parameter $T$ in (1) is assumed to be Beta distributed, the approximate expected value of $X_{1}$ up to $t^{3}$ can be obtained by using (20) as follows (note that unlike Section 2.3, the initial values are not random variables here):

$$
E\left[X_{1}\right]=\frac{1}{2}+\frac{25}{24} t-\frac{215}{192} t^{2}-\frac{9625}{2304} t^{3}+\cdots
$$

Using Laplace-Padé on (27) gives

$$
\begin{gathered}
M_{7}=-\frac{1}{1357422} e^{\left(\frac{1175}{508} t\right)}\left(-678711 \cos \left(\frac{1}{508} \sqrt{2262370} t\right)\right. \\
\left.+35 \sqrt{2262370} \sin \left(\frac{1}{508} \sqrt{2262370} t\right)\right) .
\end{gathered}
$$

Similarly, the expected value of the analytical solution $X_{2}$ becomes

$$
E\left[X_{2}\right]=\frac{1}{2} t+\frac{25}{48} t^{2}-\frac{215}{576} t^{3}+\cdots
$$

Its modification is obtained as

$$
M_{8}=\frac{4}{165} \sqrt{55} e^{\left(\frac{25 t}{24}\right)} \sin \left(\frac{3}{8} \sqrt{55} t\right)
$$

\section{THREE DIMENSIONAL ZEEMAN MODEL}

Let the initial conditions $Y_{1}, Y_{2}, Y_{3}$ be normal distributed and the model parameters $\xi, \mu, \theta$ be (standard) Beta distributed random variables. Consider the three dimensional model (Zeeman, 1977)

$$
\begin{gathered}
\frac{d x_{1}}{d t}=-x_{1}^{3}-x_{2} x_{1}-x_{3} \\
\frac{d x_{2}}{d t}=-\xi x_{1}-\mu x_{2} \\
\frac{d x_{3}}{d t}=-x_{2}-\theta \\
x_{1}(0)=Y_{1}, x_{2}(0)=Y_{2}, x_{3}(0)=Y_{3}
\end{gathered}
$$

Since the initial conditions are independent and identically distributed, $E\left[Y_{1}\right]=E\left[Y_{2}\right]=E\left[Y_{3}\right]$ as in (19) and (20). The deterministic values are given as $\xi=2, \mu=2, \theta=1$ (Zeeman, 1977). We use $\xi, \mu, \theta \sim \operatorname{Beta}(\alpha, \beta)$ where $\alpha=1$ and $\beta=1$. Using the properties of Beta distribution and (20), we get

$$
\begin{aligned}
& E[\xi]=E[\mu]=E[\theta]=\frac{\alpha}{\alpha+\beta}=\frac{1}{2} \\
& E\left[\xi^{2}\right]=E\left[\mu^{2}\right]=E\left[\theta^{2}\right]=\frac{\alpha(\alpha+1)}{(\alpha+\beta+1)(\alpha+\beta)}=\frac{1}{3}
\end{aligned}
$$




$$
\begin{aligned}
& E\left[\xi^{3}\right]=E\left[\mu^{3}\right]=E\left[\theta^{3}\right]=\frac{\alpha(\alpha+1)(\alpha+2)}{(\alpha+\beta+2)(\alpha+\beta+1)(\alpha+\beta)}=\frac{1}{4} \\
& E\left[\xi^{4}\right]=E\left[\mu^{4}\right]=E\left[\theta^{4}\right]=\frac{\alpha(\alpha+1)(\alpha+2)(\alpha+3)}{(\alpha+\beta)(\alpha+\beta+1)(\alpha+\beta+2)(\alpha+\beta+3)}=\frac{1}{5}
\end{aligned}
$$

Using this information, the expected values of $X_{1}, X_{2}$ and $X_{3}$ are obtained as below:

$$
\begin{aligned}
E\left[X_{1}\right]=E\left[Y_{1}+\right. & \left(-Y_{1} Y_{2}-Y_{1}^{3}-Y_{3}\right) t \\
& +\left(\frac{1}{2} Y_{1} Y_{2} \mu+2 Y_{1}^{3} Y_{2}+\frac{3}{2} Y_{1}^{2} Y_{3}+\frac{1}{2} Y_{1} Y_{2}^{2}+\frac{1}{2} Y_{2} Y_{3}+\frac{1}{2} Y_{1}^{2} \xi+\frac{3}{2} Y_{1}^{5}+\frac{1}{2} Y_{2}\right. \\
& \left.+\frac{1}{2} \theta\right) t^{2} \\
& +\left(-3 Y_{1}^{2} Y_{2} Y_{3}-\frac{5}{6} Y_{1}^{3} Y_{2} \mu-\frac{2}{3} Y_{1}^{2} Y_{2} \xi-\frac{1}{2} Y_{1} Y_{2}^{2} \mu-\frac{1}{2} Y_{1} Y_{3} \xi-\frac{1}{6} Y_{1}^{2} \mu \xi\right. \\
& -\frac{1}{6} Y_{1} Y_{2} \mu^{2}-\frac{1}{3} Y_{2} Y_{3} \mu-\frac{1}{6} Y_{1} Y_{2}^{3}-\frac{1}{6} Y_{2}^{2} Y_{3}-\frac{1}{6} Y_{2} \theta-Y_{1} Y_{3}^{2}-\frac{9}{2} Y_{1}^{5} Y_{2} \\
& -\frac{7}{2} Y_{1}^{4} Y_{3}-\frac{13}{6} Y_{1}^{3} Y_{2}^{2}-Y_{1}^{4} \xi-\frac{1}{2} Y_{1}^{2} Y_{2}-\frac{1}{2} Y_{1}^{2} \theta-\frac{1}{6} Y_{1} \xi-\frac{1}{6} Y_{2} \mu-\frac{5}{7} Y_{1}^{7} \\
& \left.\left.-\frac{1}{6} Y_{2}^{2}\right) t^{3}\right]+\cdots=1-6 t+53 t^{2}-\frac{27677}{36} t^{3}+\cdots
\end{aligned}
$$

Using Laplace-Padé for the truncated approximation (30) gives

$$
\begin{gathered}
M_{9}=\frac{1}{403622041} e^{\left(-\frac{23861}{840} t\right)\left(403622041 \cosh \left(\frac{1}{840} t \sqrt{403622041}\right)\right.} \\
\left.+18821 \sqrt{403622041} \sinh \left(\frac{1}{840} t \sqrt{403622041}\right)\right) .
\end{gathered}
$$

Similarly, the expected value of the approximate analytical solution $X_{2}$ is found as

$$
\begin{aligned}
E\left[X_{2}\right]=E\left[Y_{2}+\right. & \left(-Y_{1} \xi-Y_{2} \mu\right) t+\left(\frac{1}{2} Y_{1}^{3} \xi+\frac{1}{2} Y_{1} Y_{2} \xi+\frac{1}{2} Y_{3} \xi+\frac{1}{2} Y_{1} \mu \xi+\frac{1}{2} Y_{2} \mu^{2}\right) t^{2} \\
& +\left(-\frac{1}{2} Y_{1}^{5} \xi-\frac{2}{3} Y_{1}^{3} Y_{2} \xi-\frac{1}{2} Y_{1}^{2} Y_{3} \xi-\frac{1}{6} Y_{1} Y_{2}^{2} \xi-\frac{1}{6} Y_{2} Y_{3} \xi-\frac{1}{6} Y_{1}^{2} \xi^{2}\right. \\
& \left.\left.-\frac{1}{3} Y_{1} Y_{2} \mu \xi-\frac{1}{6} Y_{2} \xi-\frac{1}{6} \xi \theta-\frac{1}{6} Y_{1}^{3} \xi \mu-\frac{1}{6} Y_{3} \xi \mu-\frac{1}{6} Y_{1} \xi \mu^{2}-\frac{1}{6} Y_{2} \mu^{3}\right) t^{3}\right] \\
& +\cdots \\
\Rightarrow E\left[X_{2}\right]=1-t & +\frac{43}{24} t^{2}-\frac{661}{72} t^{3}+\cdots
\end{aligned}
$$

Using Laplace-Padé method, the modification is obtained as

$$
\begin{array}{r}
M_{10}=\frac{1}{957199} e^{\left(-\frac{309}{31} t\right)}\left(957199 \cosh \left(\frac{1}{186} t \sqrt{2871597}\right)\right. \\
\left.+556 \sqrt{2871597} \sinh \left(\frac{1}{186} t \sqrt{2871597}\right)\right)
\end{array}
$$


The expected value of $X_{3}$ is found as

$$
\begin{aligned}
& E\left[X_{3}\right]=E\left[Y_{3}+\left(-Y_{2}-\theta\right) t+\left(\frac{1}{2} Y_{1} \xi+\frac{1}{2} Y_{2} \mu\right) t^{2}\right. \\
& \left.+\left(-\frac{1}{6} Y_{1}^{3} \xi-\frac{1}{6} Y_{1} Y_{2} \xi-\frac{1}{6} Y_{3} \xi-\frac{1}{6} Y_{1} \mu \xi-\frac{1}{6} Y_{2} \mu^{2}\right) t^{3}\right]+\cdots \\
& \Rightarrow E\left[X_{3}\right]=1-\frac{3}{2} t+\frac{1}{2} t^{2}-\frac{43}{72} t^{3}+\cdots
\end{aligned}
$$

The Laplace-Padé modification of (32) becomes

$$
M_{11}=-\frac{1}{151} e^{\left(\frac{5}{6} t\right)}\left(-151 \cosh \left(\frac{1}{6} t \sqrt{151}\right)+14 \sqrt{151} \sinh \left(\frac{1}{6} t \sqrt{151}\right)\right) .
$$

\section{CONCLUSION}

In this study, we have used the random version of the Differential Transformation Method to investigate the approximate solutions of the two and three dimensional Zeeman models. The initial values $Y_{0}, Y_{1}$ and the parameter $T$ were assumed to be normal and standard Beta distributed random variables, respectively. Using these random values, the expected values and variances were found for the two dimensional model. Similarly, the same approximate characteristics were investigated for the three dimensional model too. Finally, Laplace-Padé method was used to modify these approximations and obtain new functions for the approximate numerical characteristics. It is known from the literature that the modified approximations obtained by Laplace-Padé method are generally convergent to the exact solutions in a wider time interval. As the results show, it is seen that the modified approximate results are convergent to the exact solution, which are also consistent with the deterministic results in the literature. Results from Zeeman's studies [3-5] and other studies which do not contain random components show that the systems (1), (28) successfully model the contraction and relaxation of the heart muscles and the electrical signal transmission process. In this study, we have shown the use of random components in these models shows that similar results for the biological significance of the equation systems can be achieved with additional results for the variance and standard deviations of the results that imply deviations in the muscle and electrical signal transmission behavior. This study underlines the adaptability of DTM and Laplace-Pade methods to heartbeat models with random components and the applications can also be generalized to other mathematical models.

\section{REFERENCES}

[1] Merdan M. and Khaniyev T., On the Behavior of Solutions Under the Influence of Stochastic Effect of Avian-Human Influenza Epidemic Model, International Journal of Biotechnology and Biochemistry, 4-1 (2008) 75-100.

[2] Merdan M., Bekiryazici Z., Kesemen T. and Khaniyev T., Comparison of stochastic and random models for bacterial resistance. Advances in Difference Equations, 2017-1 (2017) 133.

[3] Zeeman E.C., Differential Equations for the Heartbeat and Nerve Impulse, Mathematics Institute, University of Warwick, Coventry, UK, 1972.

[4] Zeeman E.C., Catastrophe Theory, Selected Papers 1972-1977, Addison-Wesley, Reading, MA, 1977. 
[5] Zeeman E.C., Differential equations for the heartbeat and nerve impulse, In: Waddington, C.H. (Ed.), Towards a Theoretical Biology 4: Essays, 8-67, Edinburgh University Press, 1972.

[6] Bekiryazici Z., Merdan M., Kesemen T. and Khaniyev T., Mathematical Modeling of Biochemical Reactions under Random Effects, Turkish Journal of Mathematics and Computer Science, 5 (2016) 8-18.

[7] Zhou J.K., Differential Transformation and its Applications for Electrical Circuits, Huarjung University Press, Wuuhahn, China, 1986.

[8] Ev Pukhov G.G., Differential Transforms and Circuit theory, Int. J. Circuit Theory Appl., 10-3 (1982) 265-276.

[9] Forbes C., Evans M., Hastings N. and Peacock B., Statistical Distributions, 4th Edition, John Wiley \& Sons, New Jersey, 2011.

[10] Villafuerte L. and Chen-Charpentier B.M., A random differential transform method: Theory and applications, Applied Mathematics Letters, 25-10 (2012) 1490-1494.

[11] Khudair A.R., Haddad S.A.M. and Khalaf S.L., Mean square solutions of second-order random differential equations by using the differential transformation method, Open Journal of Applied Sciences, 6-4 (2016) 287-297.

[12] Villafuerte L. and Cortés J.-C., Solving Random Differential Equations by Means of Differential Transform Methods, Advances in Dynamical Systems and Applications, 8-2 (2013) 413-425.

[13] Calbo G., Cortés J.-C., Jódar L. and Villafuerte L., Solving the random Legendre differential equation: mean square power series solution and its statistical functions, Comput. Math. Appl., 61-9 (2011) 2782-2792.

[14] Villafuerte L., Braumann C.A., Cortés J.-C. and Jódar L., Random differential operational calculus: Theory and applications, Computers and Mathematics with Applications, 59-1 (2010) 115-125.

[15] Gökdoğan A., Merdan M. and Yildirim A., The Modified Algorithm for the Differential Transform Method to Solution of Genesio Systems, Communications in Nonlinear Science and Numerical Simulation, 17-1 (2012) 45-51.

[16] Benhammouda B. and Vazquez-Leal H., Analytical solutions for systems of partial differentialalgebraic equations, SpringerPlus, 3-1 (2014) 137.

[17] Sungu, I.C. and Demir, H., A Computational Method for the Time-Fractional Navier-Stokes Equation, Cumhuriyet Science Journal, 39-4 (2018) 900-911.

[18] Merdan, M., Bekiryazici, Z., Kesemen, T. and Khaniyev, T., Analyzing the dynamics of Ebola transmission with random effects. Communications in Mathematical Biology and Neuroscience, 2018 (2018) Article-ID 22.

[19] Mehdi Rashidi, M. andErfani, E., The modified differential transform method for investigating nano boundary-layers over stretching surfaces. International Journal of Numerical Methods for Heat \& Fluid Flow, 21-7 (2011) 864-883.

[20] Onwubuoya, C., Nwanze, D. E., Erejuwa, J. S. and Akinyemi, S. T., An Approximate Solution of a Computer Virus Model with Antivirus using Modifed Differential Transform Method, International Journal of Engineering Research \& Technology, 7-4 (2018) 154-161.

[21] Bekiryazici, Z., Bazi Kompartmanli Modellerin Rastgele Ektiler Altinda Incelenmesi, PhD Thesis, Karadeniz Technical University, Trabzon, 2017. 